\title{
Spatial frequency domain imaging: a quantitative, noninvasive tool for in vivo monitoring of burn wound and skin graft healing
}

Gordon T. Kennedy

Randolph Stone, II

Andrew C. Kowalczewski

Rebecca Rowland

Jeffrey H. Chen

Melissa L. Baldado

Adrien Ponticorvo

Nicole Bernal

Robert J. Christy

Anthony J. Durkin 


\title{
Spatial frequency domain imaging: a quantitative, noninvasive tool for in vivo monitoring of burn wound and skin graft healing
}

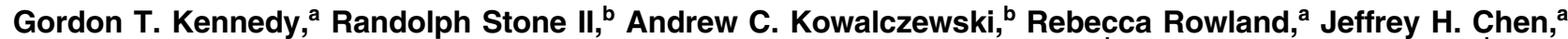 \\ Melissa L. Baldado, ${ }^{a}$ Adrien Ponticorvo, ${ }^{a}$ Nicole Bernal, ${ }^{c}$ Robert J. Christy, ${ }^{b}$ and Anthony J. Durkin ${ }^{a, d, *}$ \\ aUniversity of California, Beckman Laser Institute and Medical Clinic, Irvine, California, United States \\ ${ }^{b}$ Combat Trauma and Burn Injury Research, United States Army Institute of Surgical Research, JBSA Fort Sam Houston, Texas, United States \\ 'UC Irvine Regional Burn Center, Department of Surgery, Orange, California, United States \\ dUniversity of California, Department of Biomedical Engineering, Irvine, California, United States
}

\begin{abstract}
There is a need for noninvasive, quantitative methods to characterize wound healing in the context of longitudinal investigations related to regenerative medicine. Such tools have the potential to inform the assessment of wound status and healing progression and aid the development of new treatments. We employed spatial frequency domain imaging (SFDI) to characterize the changes in optical properties of tissue during wound healing progression in a porcine model of split-thickness skin grafts and also in a model of burn wound healing with no graft intervention. Changes in the reduced scattering coefficient measured using SFDI correlated with structural changes reported by histology of biopsies taken concurrently. SFDI was able to measure spatial inhomogeneity in the wounds and predicted heterogeneous healing. In addition, we were able to visualize differences in healing rate, depending on whether a wound was debrided and grafted, versus not debrided and left to heal without intervention apart from topical burn wound care. Changes in the concentration of oxy- and deoxyhemoglobin were also quantified, giving insight into hemodynamic changes during healing. ๑ The Authors. Published by SPIE under a Creative Commons Attribution 4.0 Unported License. Distribution or reproduction of this work in whole or in part requires full attribution of the original publication, including its DOI. [DOI: 10.1117/1.JBO.24.7.071615]
\end{abstract}

Keywords: spatial frequency domain imaging; diffuse optics; multispectral imaging; graft viability; wound healing; burns.

Paper 180582SSRR received Oct. 12, 2018; accepted for publication Jun. 27, 2019; published online Jul. 16, 2019.

\section{Background}

Annually in the USA, there are $\sim 500,000$ burn wounds that require medical attention and, of these, some 40,000 patients require hospitalization for their burn injuries. ${ }^{1}$ A large number of these patients will require skin grafts. For deep partial to full thickness burns, the standard of care is surgical debridement of the devitalized burned tissue, through a technique called tangential excision, followed by application of an autologous splitthickness skin graft. ${ }^{2}$ In this procedure, the patient's own skin, consisting of the epidermis and a portion of dermis, is taken from a donor site using a dermatome. The autograft may be meshed, allowing it to be expanded to cover a greater surface area. Typically the donor wounds re-epithelialize in 10 to 14 days and the autografted burns will accept a graft by day 5 and become fixed and durable in 10 to 14 days. In many cases, the grafted burn heals with some degree of contraction and scarring, depending on the degree of the meshing, the depth of burn, and the timeliness of accurate burn wound assessment and surgical intervention. ${ }^{3}$

Further innovation in burn wound healing outcomes research is being carried out by various groups with respect to new treatment methods that include amalgams of engineered tissue constructs, adipose derived stem cells, and growth factors. ${ }^{4}$ These have the potential to reduce graft failure, promote faster healing, result in better cosmetic outcomes, and enable grafting with less autologous donor skin.

\footnotetext{
*Address all correspondence to Anthony J. Durkin, E-mail: adurkin @uci.edu
}

A variety of animal models have been employed to test and evaluate these new therapies, with the most common being a porcine model employing Yorkshire or Red Duroc pigs. The gold standard for assessing the status of the wound related to the process of skin graft healing is the histopathologic examination of biopsy tissue. Although this offers micron-scale resolution with a large number of stains that can highlight the different structures and molecular processes in the skin, the biopsy is an invasive procedure that perturbs the surrounding tissue. It provides only a snapshot of a small region of tissue at the time and location that the biopsy was harvested. In addition, the biopsy site and adjacent tissue become unusable. This makes longitudinal studies cumbersome due to the requirement of many homogeneous treatments over numerous animals that may span up to 120 days, depending on the specific phase of healing that is under investigation.

Previously, we have investigated spatial frequency domain imaging (SFDI) for noninvasively determining the severity of burn wounds. ${ }^{5}$ Using a porcine model of graded burn wounds, we showed that the reduced scattering coefficient was correlated with burn severity and that these characteristic changes in the reduced scattering can be measured within hours of the occurrence of a burn. In this paper, we examine the potential for SFDI to noninvasively assess the healing status of burn wounds and skin grafts. As far as the authors are aware, at the time of this writing, the measurement of tissue optical properties by SFDI for long-term healing has not been applied to skin graft healing. SFDI has been applied to characterize burn severity. ${ }^{5-10}$ Additionally, in preclinical burn wound models, SFDI has been applied to the evaluation of infection ${ }^{11}$ and hypertrophic 
scarring, ${ }^{12}$ and diffuse reflectance spectroscopy has been applied to the monitoring of tissue constructs. ${ }^{13}$ Porcine skin optical properties measured in this work broadly agree with those previously reported.

SFDI is a wide-field diffuse optical imaging technique that enables noninvasive quantitative determination of the reduced scattering and absorption coefficients of in vivo tissue. The optical property results are an average over a depth of $\sim 5 \mathrm{~mm} .{ }^{14}$ The reduced scattering coefficient provides a measure of the structure of the skin (predominantly collagen and elastin matrix), and the ability to quantify this parameter over a wide field is unique to SFDI. ${ }^{9}$ The absorption coefficient spectrum enables the determination of chromophore concentrations such as oxy- and deoxyhemoglobin, total hemoglobin, oxygen saturation, and water fraction and is thus a measure of the physiological state of the tissue.

Here we extend that work and demonstrate that SFDI can be used to measure structural changes in the wound bed during longitudinal healing investigations. We correlate these changes in structure, as reported via reduced scattering coefficient, to changes in tissue microstructure as reported by histology. In the first set of studies, we employ SFDI to determine characteristic changes that occur in burn wound healing in the absence of intervention (no graft or debridement). Thereafter, we use SFDI to quantify the reduced scattering and absorption coefficients, oxy- and deoxyhemoglobin concentrations, and tissue oxygen saturation, which are related to the healing of surgically debrided wounds with and without split-thickness skin grafts.

\section{Methods}

\subsection{Spatial Frequency Domain Imaging}

For these studies, we employed a commercially available SFDI measurement system capable of imaging optical properties (absorption coefficient $\mu_{a}$ and reduced scattering coefficient $\left.\mu_{s}^{\prime}\right)^{15}$ of tissue over large fields of view $(20 \times 15 \mathrm{~cm})$ (ReflectRS ${ }^{\circledR}$, Modulated Imaging, Inc., Irvine, California). The device was placed at a height of $32 \mathrm{~cm}$ above each tissue region to be imaged. The device was controlled using MI Acquire v1.34.00 software that accompanies the instrument. Each region of interest was imaged three consecutive times requiring a total of $\sim 90 \mathrm{~s}$. Sinusoidal patterns are projected and images were captured at 8 wavelengths between 471 and $850 \mathrm{~nm}$ and at five spatial frequencies evenly spaced between 0 and $0.2 \mathrm{~mm}^{-1}$ according to a protocol that we have previously employed. ${ }^{9}$

Imaging was carried out immediately before burns were created, immediately after burns, and then periodically over the duration of each investigation (28 days for wound healing in the absence of intervention and 60 days for skin graft healing).

After each imaging session, a reference calibration phantom having known optical properties (provided with the commercial SFDI instrument) was measured using the SFDI device under the same lighting conditions as the animal measurements, as has been previously described in detail. ${ }^{14}$ This phantom is made of a silicone-based polymer, mixed with India ink and titanium dioxide, to mimic tissue optical properties. ${ }^{16}$ After all SFDI measurements were completed, the raw image files were processed using the MI Analysis software package (Modulated Imaging, Inc., Irvine, California). Through this processing, the reflectance images were calibrated against the reference phantom measurements and converted to images of the absorption $\left(\mu_{a}\right)$ and reduced scattering coefficients $\left(\mu_{s}^{\prime}\right)$ at all measured wavelengths.
The SFDI data were corrected for both height variations from -20 to $+20 \mathrm{~mm}$ and angles of $\pm 30 \mathrm{deg}$, and Gaussian smoothing (7 pixel kernel) was employed. A schematic of the imaging setup and processing workflow is depicted in Fig. 1.

\subsection{Burn Wound Healing in the Absence of Intervention}

We have previously described the porcine model of graded burn wound severity in detail. ${ }^{9}$ SFDI data were acquired immediately before and again within an hour after burn wounds were created as described in the previous section. These studies were carried out under UCI animal care and use protocol \#2015-3014. Briefly, anesthetized Yorkshire pigs $(N=6)$ received burns $(N=16$ per animal) of $3-\mathrm{cm}$ diameter to either side of the dorsal line defined by the spine using a heated brass burn cylinder. The duration of contact between brass and skin was varied between 5 and $40 \mathrm{~s}$ in 5-s intervals, enabling a range of burn severities from superficial to full thickness. After imaging, a topical antibiotic was applied and the wounds were dressed. On days 7, 14, 21, and 28 postburn, the dressings were removed and the wounds were reimaged. Punch biopsies (6-mm diameter) were taken for histological evaluation using Masson's trichrome to map the extent of thermal damage and collagen status. Images and biopsies were also taken from a comparable region of healthy, unburned skin at each time point as control measurements. The biopsies were taken after imaging to avoid contaminating the wound site with blood. Additionally, biopsy sites were excluded from SFDI ROI selection after the biopsies were taken, as the resulting biopsy wounds are not representative of the burn tissue. A schematic of the burn and biopsy locations and timeline for the measurements is presented as Fig. 2(a).

\subsection{Wound Healing Following Split-Thickness Skin Grafts}

These studies were carried out under an US Army Institute for Surgical Research approved IACUC protocol. Animal research was conducted in compliance with the Animal Welfare Act, the implementing Animal Welfare Regulations, and the principles of the Guide for the Care and Use of Laboratory Animals, National Research Council. The facility's Institutional Animal Care and Use Committee approved all research conducted in this study. The facility where this research was conducted is fully accredited by AAALAC International. Animals were housed individually in a temperature-controlled environment with a 12-h light/dark cycle in the AALAAC approved vivarium at the USAISR with access to water and food ad libitum. Before burning, hair was removed from the dorsum of the swine with clippers, and the skin was sterilized with chlorohexidine. Ten deep partial thickness (DPT) burns were administered to the dorsum of anesthetized Yorkshire female swine using a $5 \times 5 \mathrm{~cm}$ brass burn tool with active temperature control ${ }^{17}$ that was set to $100^{\circ} \mathrm{C}$. Prior to burning, the perimeter of each region was tattooed with an electric tattoo machine (Spaulding and Rogers, Voorhesville, New York) to enable ease of visualization of changes in the wound shape and size due to growth and wound contraction. Four days after the burn, the wounds were surgically debrided with a scalpel to a bleeding wound bed. A split-thickness skin graft was harvested using a pneumatic dermatome (Zimmer, Warsaw, Indiana) at 12/1000th of an inch from the upper hindlimb area of the animal, meshed at a 1.5:1 ratio, and secured to each wound bed with staples. ${ }^{18}$ Of the 10 burn wounds, 8 


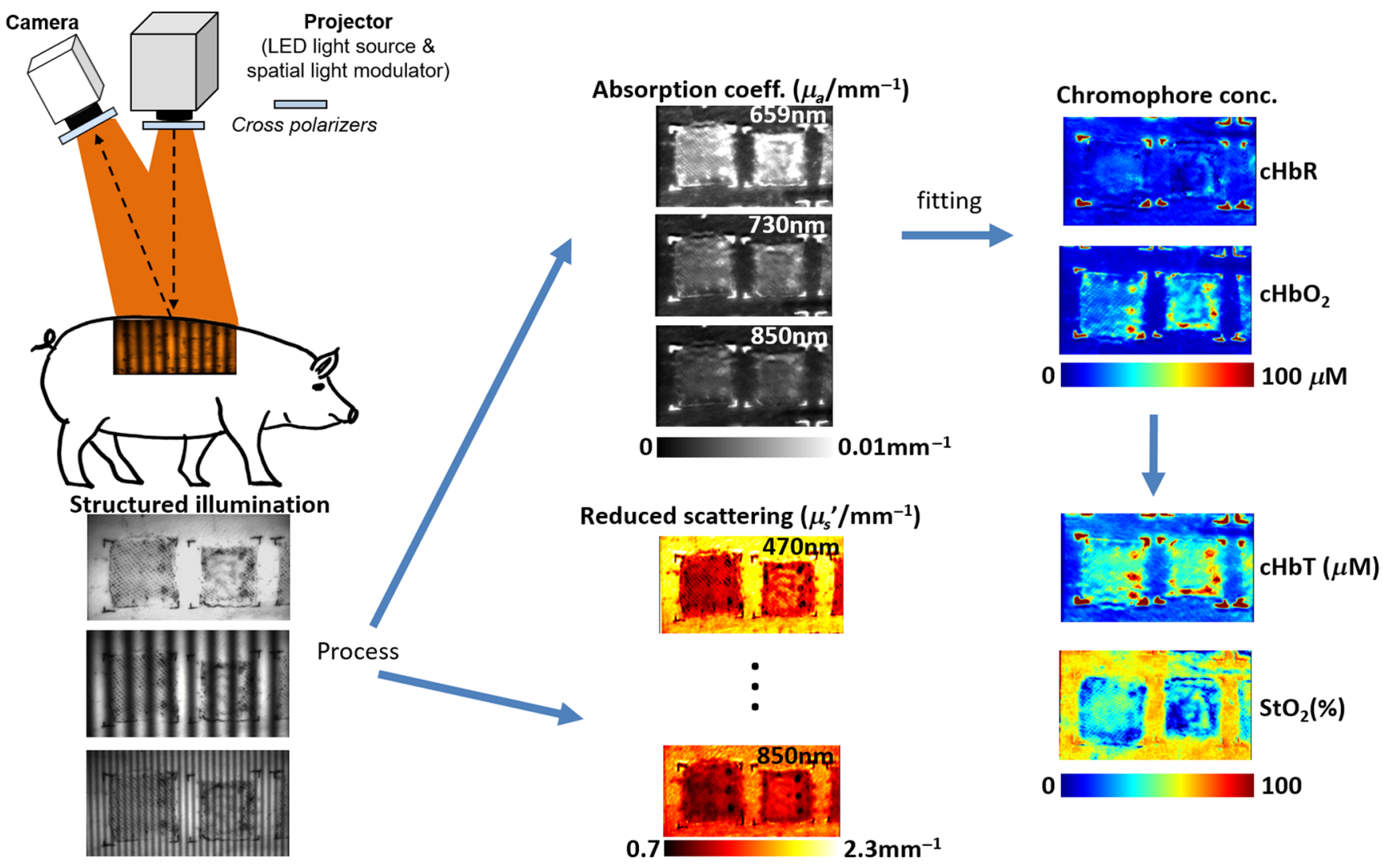

Fig. 1 SFDI configuration for measurements of in vivo porcine skin.

Burn healing - no treatment
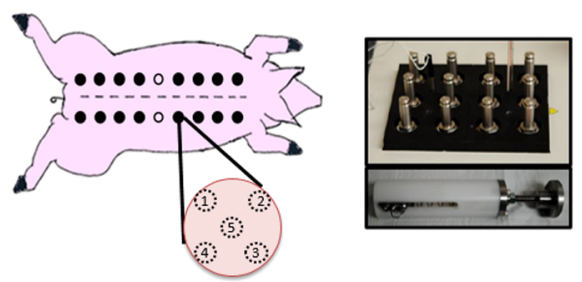

- 16 burns , $\phi 3 \mathrm{~cm}$

- 5 - 40 s contact time $(100 \mathrm{C})$

- 2 controls

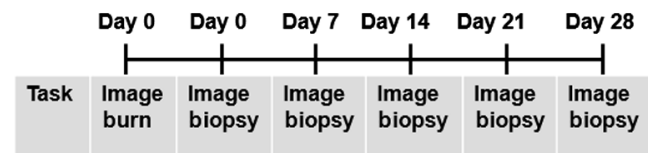

(a)
Treatment: split thickness skin graft

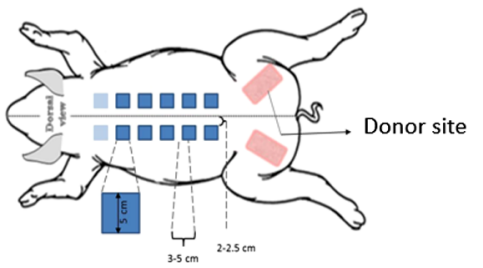

- 10 burns, $\square 5 \mathrm{~cm}$

- All deep partial thickness

- 2 controls

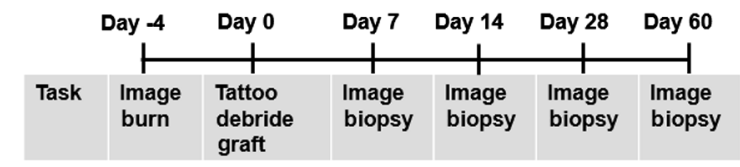

(b)

Fig. 2 Sketch of method.

received additional treatments as part of other studies and were not suitable for analysis for this work. However, one burn was debrided and treated with a split-thickness skin graft in the conventional manner and one burn wound was surgically debrided but received no treatment. Two regions consisting of unburned normal skin were used as controls. Wounds were dressed with antimicrobial Telfa, Kerlix, Coban (Covidien, Mansfield, Massachusetts), and a jacket to protect the grafted wounds from infection and mechanical shearing forces. On days 7, 14, 28, and 60 after grafting, the dressings were removed and the wounds were imaged using SFDI. Punch biopsies (8-mm diameter) were harvested after imaging from the graft sites on day 14 and 28 and a strip biopsy spanning the entire length of the wound was collected after the last measurement on day 60. These were stained using Masson's trichrome and analyzed by an histologist. A schematic of the experiment is presented as Fig. 2(b). 


\section{Results}

\subsection{Burn Wound Healing in the Absence of Intervention}

Figure 3 shows the development of the reduced scattering coefficient, measured at $659 \mathrm{~nm}$ along with color photographs of the wound for a typical superficial partial thickness (SPT) burn. This wavelength was selected as it was near the center of the wavelength range; however, the trends in reduced scattering are similar for all wavelengths. The locations of the regions of interest (ROIs) for analysis are shown in the scattering maps (magenta circle). ROI diameter was chosen to be $6 \mathrm{~mm}$ to match the diameter of the circular punch biopsies that were collected and the location of the ROIs were selected to have a reduced scattering value similar to that of the initial biopsy location used to classify the burn severity. For each burn, the ROI location was maintained for all measurement time points. Initially postburn, we measured a decrease in the magnitude of the reduced scattering coefficient, which is consistent with previously reported results, ${ }^{9}$ where the reduced scattering coefficient decreased as the burn severity increased. For SPT burns, there was a modest ( $\sim 15 \%$ to $25 \%$ ) decrease in reduced scattering coefficient that returned to a baseline level after $\sim 21$ days as is illustrated in the graph of scattering versus time in Fig. 3(c).

For DPT burns (Fig. 4), the results were more complex. Although the burns were created in a consistent manner using a burn tool having a uniform temperature, the resulting burns were spatially heterogeneous in severity. This resulted in nonuniform healing with islands of less severely burned skin that healed faster. The reduced scattering coefficient measured by SFDI was able to identify these regions and predict the healing response (as verified by histology). This has been previously described by Ponticorvo et al. ${ }^{19}$

Figure 5 illustrates the progression of healing for a typical full thickness burn that was created by 40-s contact duration with the $100^{\circ} \mathrm{C}$ burn tool. In the case of full thickness burns (red trace, Fig. 5), the scattering at the center of the burn was slightly elevated above that of unburned skin at day 7 and then decreased through day 28. The downward trend in scattering coefficient, which is attributed to the thermal denaturation of the extracellular matrix, continued as the necrotic tissue was replaced by granulation tissue. At day 28, the wound had not healed and the reduced scattering coefficient measured at $659 \mathrm{~nm}$ was $\sim 50 \%$ less than that of unburned skin. (There was also significant wound contraction.) For statistical analysis, we used data from 28 burns at each time point: (11 SPT burns having a burn duration of $5 \mathrm{~s}, 9$ DPT burns having a burn duration of $25 \mathrm{~s}$, and 8 FT burns from 40-s duration burns). Histology from biopsies taken at 24-h postburn was used to grade the burn severity (benchmark standard). Because tissue was removed periodically throughout the 28 day study for histological evaluation, the biopsy locations were not suitable for longitudinal analysis. Therefore, for each burn, a $20 \times$ 20 pixel ROI that was present throughout the entire study and had similar reduced scattering coefficient to the tissue that was histologically graded at $24 \mathrm{~h}$ was selected for analysis. For each burn, the mean reduced scattering of the ROI was calculated at each time point enabling the comparison of reduced scattering for each of the different burn severities. At day 28, the mean scattering from the SPT burns was not significantly different to surrounding unburned tissue (paired $t$-test, $p<0.05$ ), whereas the mean reduced scattering coefficients for the DPT and FT burns were significantly different than adjacent unburned tissue (paired $t$-test, $p<0.05$ ) but were not statistically different to each other.

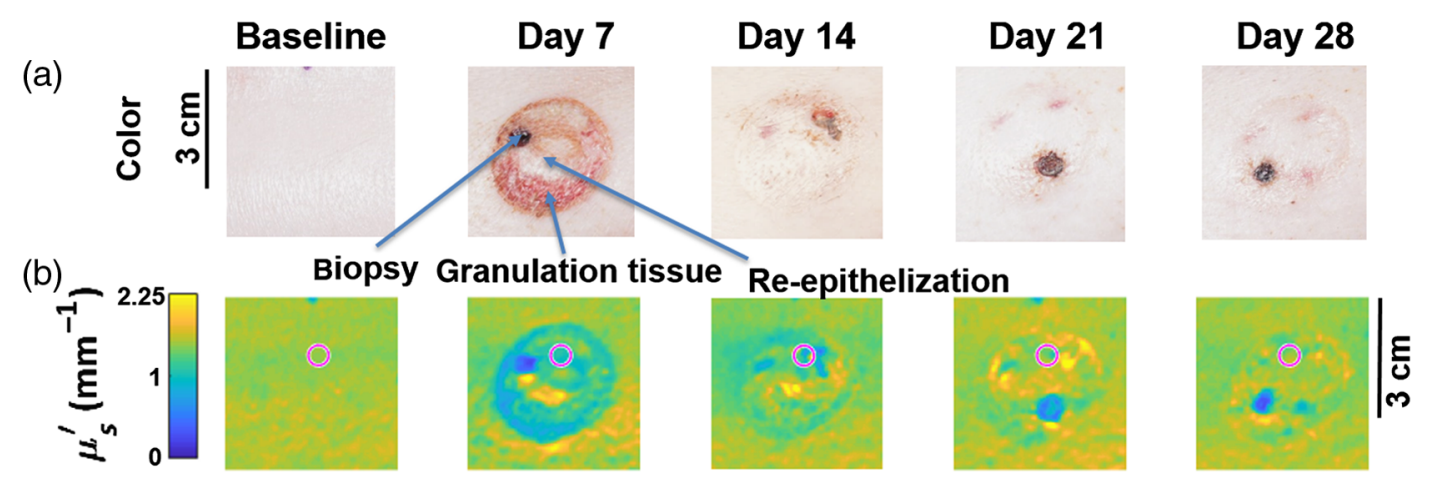

(c)

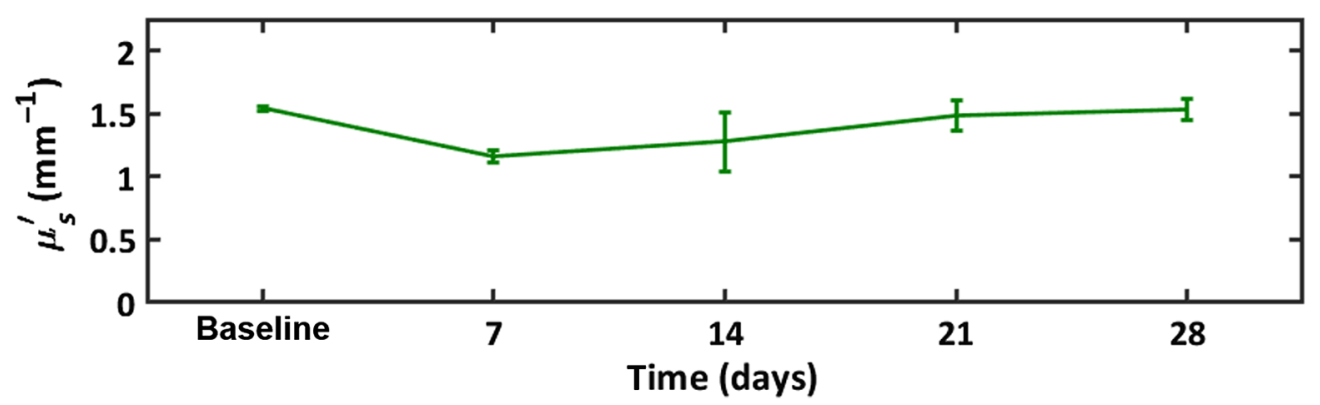

Fig. 3 Healing of a SPT burn over 28 days in the absence of intervention. (a) Color photographs of the burns. Pertinent features of the wound healing are labeled for day 7 . Biopsy locations follow the diagram in Fig. 2(a). (b) SFDI maps of the reduced scattering coefficient at $659 \mathrm{~nm}$. (c) Graph of the reduced scattering coefficient for the region of interest shown in the maps above (magenta circle). 


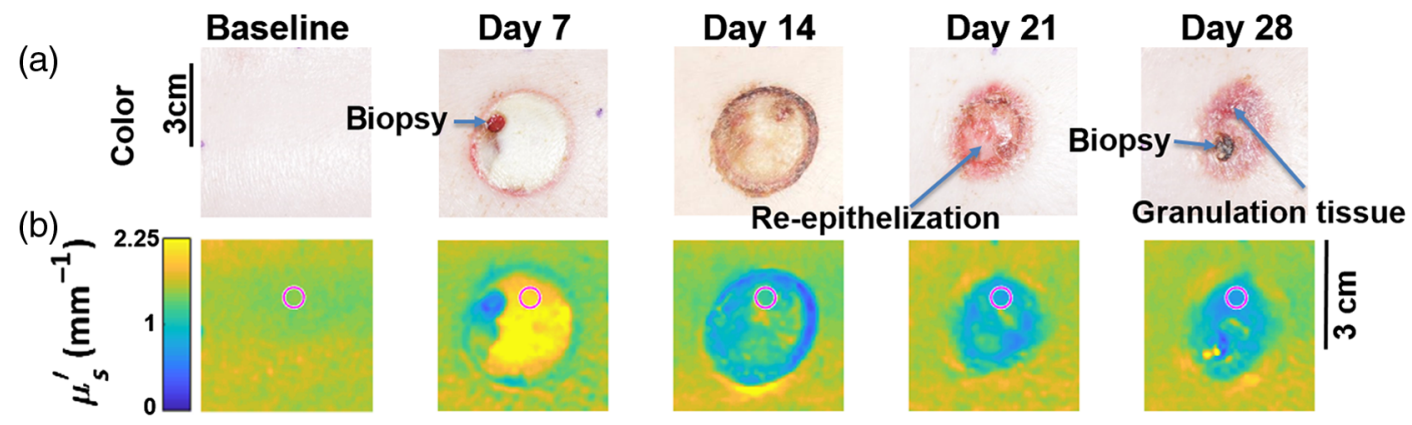

(c)

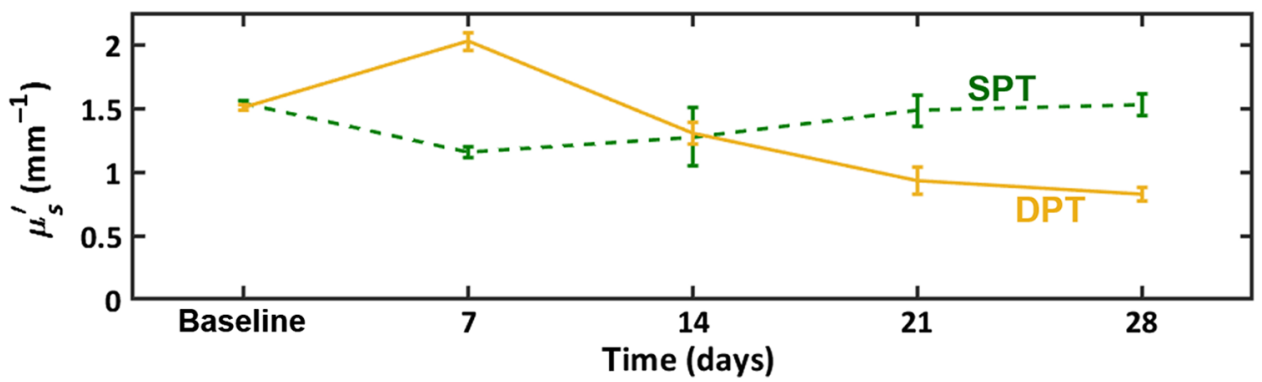

Fig. 4 Healing of a deep partial thickness burn over 28 days in the absence of intervention. (a) Color photographs of the burns. Pertinent features of the wound are labeled. Biopsy locations follow the diagram in Fig. 2(a). (b) SFDI maps of the reduced scattering coefficient at $659 \mathrm{~nm}$. (c) Mean and standard deviation reduced scattering coefficients for the ROls shown in the maps above (magenta circle). For comparison, the reduced scattering values for SPT burn (green dotted line) are also presented.

(a)

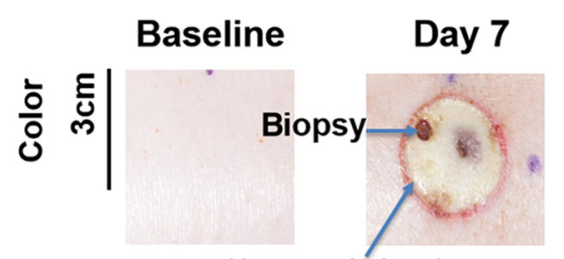

Hyperemic border
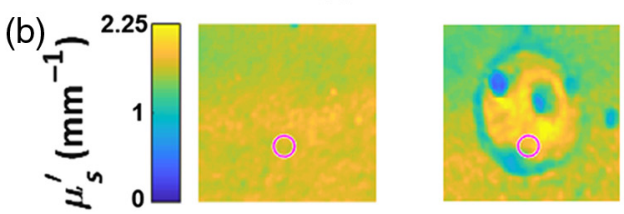

Day 14

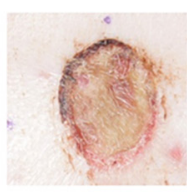

Re-epithelization from edge wound contraction

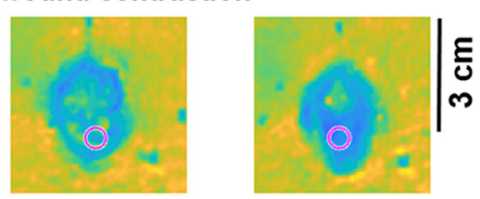

(c)

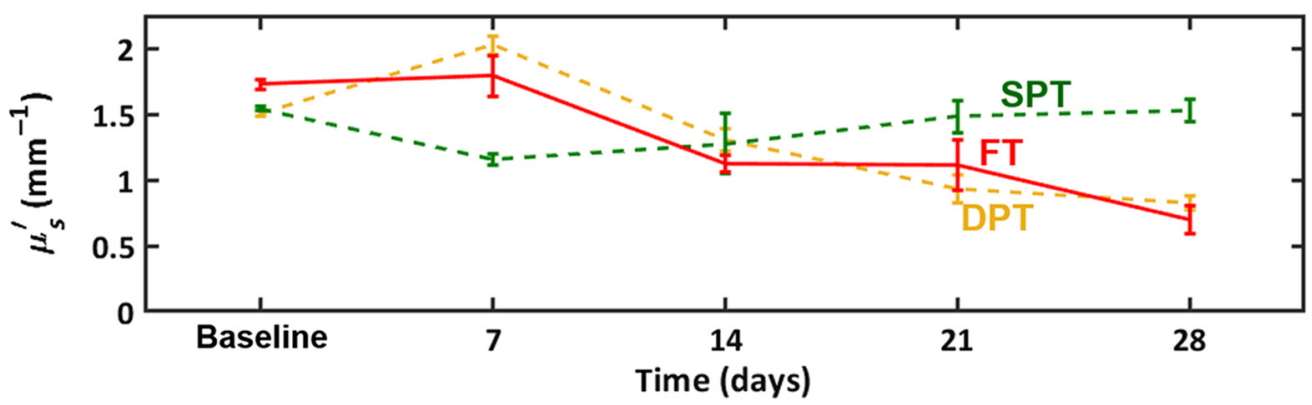

Fig. 5 Healing of a full thickness burn over 28 days in the absence of intervention. (a) Color photographs of the burns. Pertinent features of the wound are labeled. Biopsy locations follow the diagram in Fig. 2(a). (b) SFDI maps of the reduced scattering coefficient at $659 \mathrm{~nm}$. (c) Mean and standard deviation reduced scattering coefficients for the ROls shown in the maps above. For comparison, the reduced scattering values for SPT (green dotted line) and deep partial thickness burns (yellow dotted line) are also presented. 


\subsection{Wound Healing Following Split-Thickness Skin Grafts}

For the wound that was surgically debrided and not grafted (Fig. 6), we observed a dramatic decrease in reduced scattering coefficient 7 days after debridement $\left(\overline{\mu_{s}^{\prime}}=0.47 \mathrm{~mm}^{-1}\right.$, standard deviation $0.09 \mathrm{~mm}^{-1}$ at $659 \mathrm{~nm}$,) compared to that of unburned skin $\left(\overline{\mu_{s}^{\prime}}=1.62 \mathrm{~mm}^{-1}\right.$, standard deviation $\left.0.06 \mathrm{~mm}^{-1}\right)$ measured for a circular ROI having a 28 -mm diameter, centered on the burn. This is attributed to granulation tissue and low scattering of the hypodermal or adipose tissue. The depressed scattering persists for most of the wound area for the duration of the experiment, although the scattering at the periphery increases over time as the wound edges re-epithelialize and the burn area is decreased by contraction, as indicated by the hourglass shape at day 60 in Fig. 6. Color images of the surgically debrided wound progression together with false color maps of the reduced scattering coefficient at a wavelength of $659 \mathrm{~nm}$ and a plot of reduced scattering coefficient versus time for the central 28-mm ROI (magenta circle) are presented in Fig. 6.

The evolution of a typical wound that received a split-thickness skin graft from baseline (immediately before burning) to 60 days postgraft is presented in Fig. 7. At day 7, we observe a decrease in reduced scattering that is less than the decrease in the reduced scattering of the untreated excised wound bed shown in Fig. $7\left(\overline{\mu_{s}^{\prime}}=1.0 \mathrm{~mm}^{-1}\right.$, standard deviation $0.13 \mathrm{~mm}^{-1}$ versus $\overline{\mu_{s}^{\prime}}=0.47 \mathrm{~mm}^{-1}$, standard deviation $0.09 \mathrm{~mm}^{-1}$ at $659 \mathrm{~nm}$ ) as the measurement includes the skin graft, which has been applied over the wound bed. However, in this case, the reduced scattering increases uniformly across the entire wound, achieving a reduced scattering close to that of the unburned baseline measurement at day $60 \overline{\left(\mu_{s}^{\prime}\right.}=1.51 \mathrm{~mm}^{-1}$, standard deviation $0.18 \mathrm{~mm}^{-1}$ at D60 versus $\overline{\mu_{s}^{\prime}}=1.62 \mathrm{~mm}^{-1}$, standard deviation $0.07 \mathrm{~mm}^{-1}$ for baseline at a wavelength of $659 \mathrm{~nm}$ )
Kruskall-Wallis $\mathrm{H}$ tests of the reduced scattering at $659 \mathrm{~nm}$ in the central $28-\mathrm{mm}$ ROI (7825 pixels) show that the mean ranks are different for all time points $(p=0.01)$. We note that the skin grafts are clearly multilayer structures comprising two or perhaps three distinct layers-skin graft, granulation tissue consisting of a mix of immature and mature collagen, and hypodermis/adipose tissue. Although our analysis employs a semiinfinite model for calculating the tissue optical properties, it nevertheless provides a measure of the average properties of the tissue over the interrogation volume. A multilayer model may provide greater insight and is an area of current research. ${ }^{20}$

The measurements of the reduced scattering correlate with the structure revealed by Masson's trichrome histology that is presented in Fig. 7. At 14 days after grafting, a modest amount of granulation tissue has formed by deposition of immature collagen between the overlaying graft and the excised wound bed. This is evident in the histology image (day 14, Fig. 7) as the light blue labeled area. Although the wound has re-epithelialized, the granulation tissue extends to the epidermis where interstitial spaces were created by meshing the graft to cover a larger area. The wound is also approximately half the thickness of unburned skin because of the surgical debridement. At day 28, the wound site still has reduced thickness but the immature collagen in the granulation tissue is being replaced by more mature collagen (light blue to darker blue) and larger blood vessels are forming (immediately to the right of the \# in Fig. 7, D28). By day 60, the wound is thicker, approaching that of uninjured skin. In the top half of the dermis, type I mature collagen has formed while type III immature collagen extends to the base of the wound.

To investigate the relationship between healing status and reduced scattering coefficient, we examined the reduced scattering as a function of wavelength. In tissue, the monotonic decrease in scattering with increasing wavelength, combined (a)

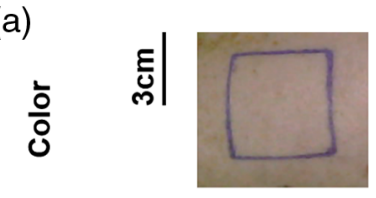

(b)

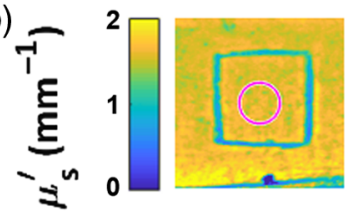

Day 7
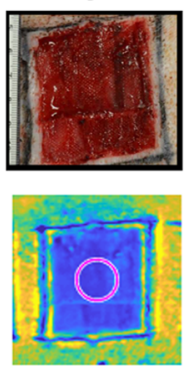

Day 14
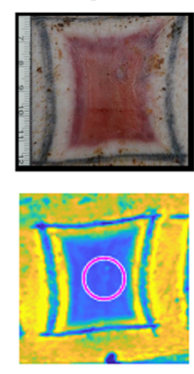

Day 28
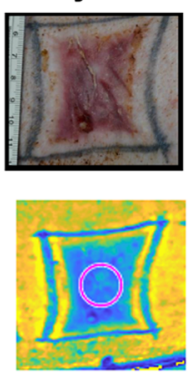

Day 60
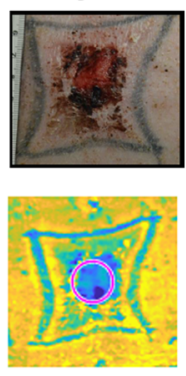

(c)

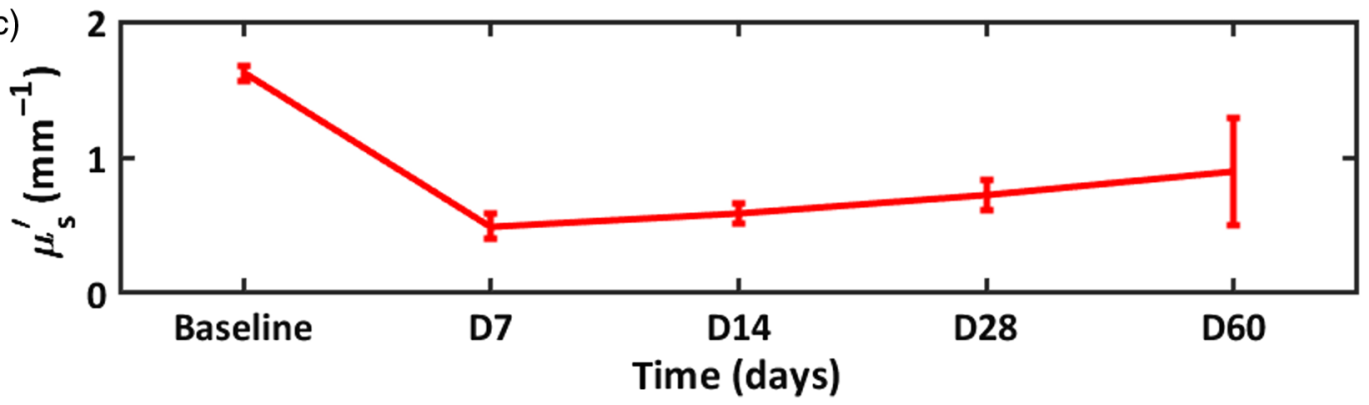

Fig. 6 Healing of an excised burn wound with no graft. (a) Color photographs documenting the time course. (b) SFDI maps of the reduced scattering coefficient at $659 \mathrm{~nm}$. (c) Graph of the reduced scattering coefficient for the central $28 \mathrm{~mm}$ diameter region of interest (magenta circle) shown in the scattering maps above. 

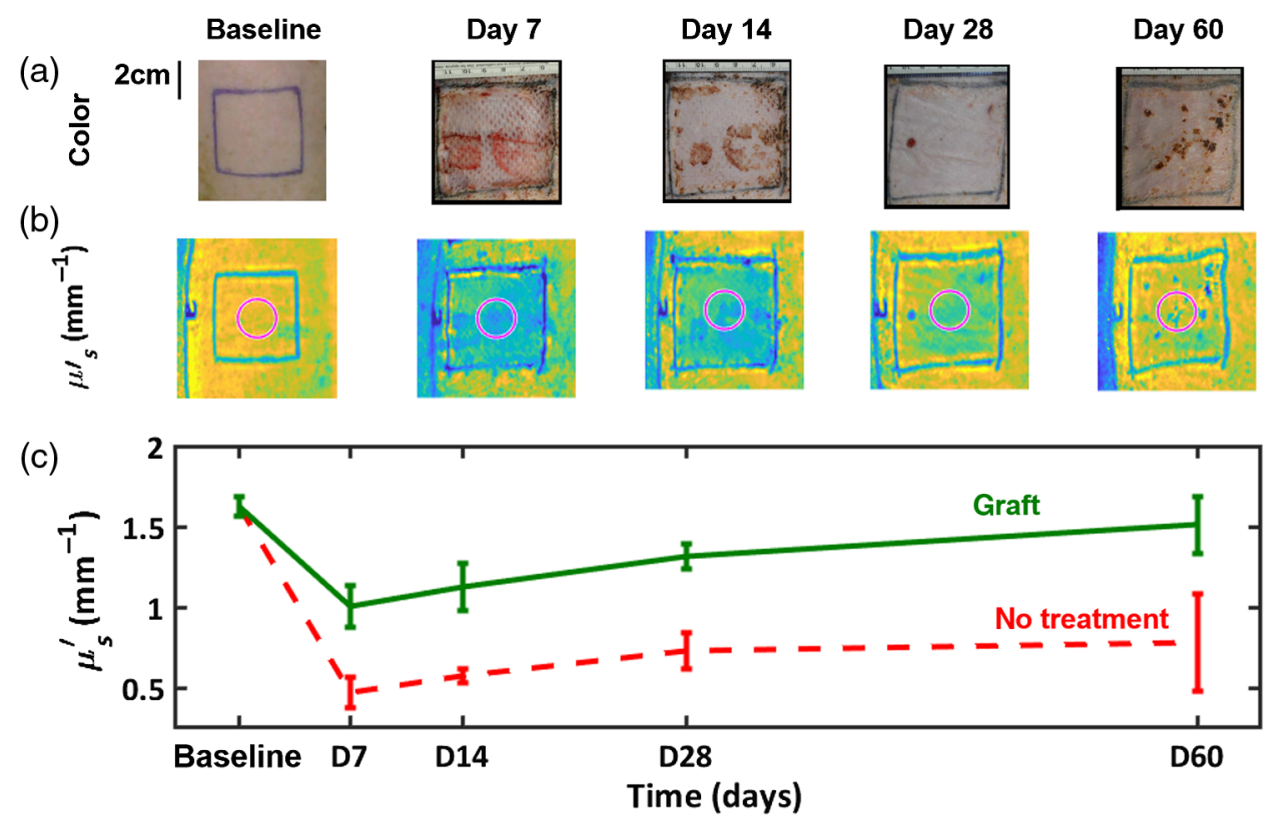

(d)
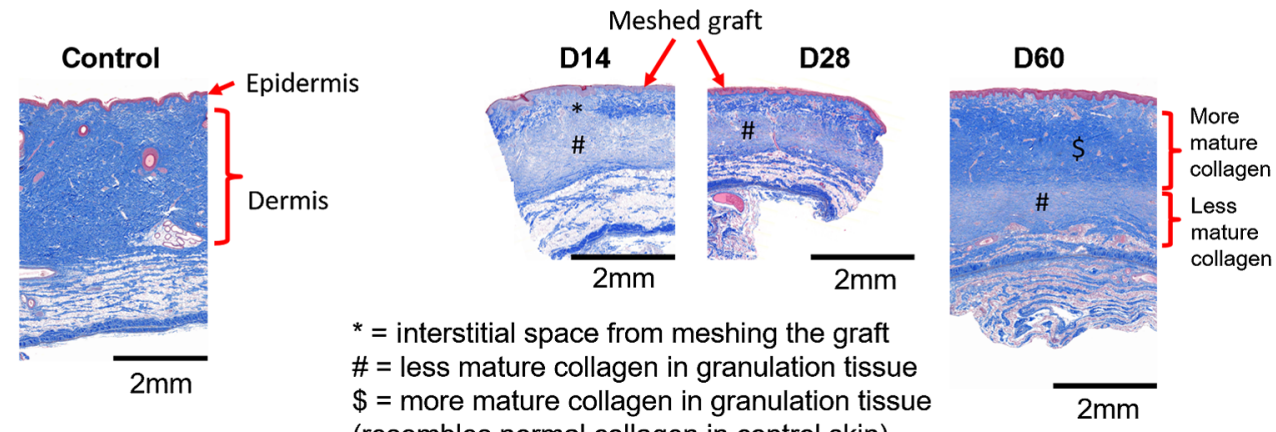

Fig. 7 Healing of a burn treated with a split-thickness skin graft. (a) Color photographs documenting the time course. (b) SFDI maps of the reduced scattering coefficient at $659 \mathrm{~nm}$. (c) Graphs of the mean and standard deviation of the reduced scattering coefficient for a central region of interest (magenta circle, diameter $28 \mathrm{~mm}$ ) shown in the scattering maps above. (d) Masson's trichrome histology of punch biopsies taken from a split-thickness skin graft at 14,28 , and 60 days after grafting. Histology of unburned control skin is also presented.

with decreasing absorption results in an increasing interrogation depth as wavelength increases from 590 to $850 \mathrm{~nm}$. Figure 8 shows a plot of the reduced scattering for the wavelengths of 591, 659, and $851 \mathrm{~nm}$, along with the penetration depth for structured light having a spatial frequency of $0.1 \mathrm{~mm}^{-1}$ superimposed on the histology image at D60. The scattering values have been normalized to their baseline (preburn) values to aid comparison. As wavelength increases, we observe a smaller recovery of the reduced scattering compared to the preburn values. We attribute this to deeper penetration of these wavelengths into the tissue where there is less mature collagen and more granulation tissue that has lower reduced scattering. For the longest wavelengths and early time points after the skin graft procedure, the wound bed is shallow and the SFDI may also be interrogating the hypodermal fat. Interestingly, at $591 \mathrm{~nm}$, we observe that the reduced scattering at days 28 and 60 increased to a value greater than that measured before the burn. This may be due to greater scattering from the epidermis and papillary dermis. Histology shows the epidermis of the grafted regions to be thicker than control skin at days 28 and 60 .

Since SFDI is a wide-field imaging technique, we are able to spatially resolve the optical properties over the entire wound area. Figure 9 illustrates the two-dimensional map of reduced scattering coefficient at day 60 for a burn that was only surgically debrided and a burn that received a meshed split-thickness skin graft. Cross sections of the reduced scattering coefficient at a wavelength of $659 \mathrm{~nm}$ through the central region of the wounds are presented beneath the spatial maps along with images of the histology obtained from strip biopsies that traverse the length of the healing wound. For the wound without a graft, the scattering maps depict healing from the edge toward the center as the wound re-epithelializes from the periphery and fibroblasts migrate into the wound and deposit new collagen. This is supported by the histology, which shows a thick region of granulation tissue in the center of the wound. This becomes thinner toward that of normal skin at the edge. Evidence of wound contraction is also clearly present (the dips in scattering at the edge of the wound are artifacts due to the tattoos that were applied around the burns for tracking their shape and size). In contrast, the grafted wound [Fig. 9(b)] healed in a more uniform manner, as expected. By day 60, the reduced scattering approaches that of normal skin as can be seen in the color map of the reduced scattering coefficient, which contains adjacent regions outside the wound area. The homogeneity of the reduced 

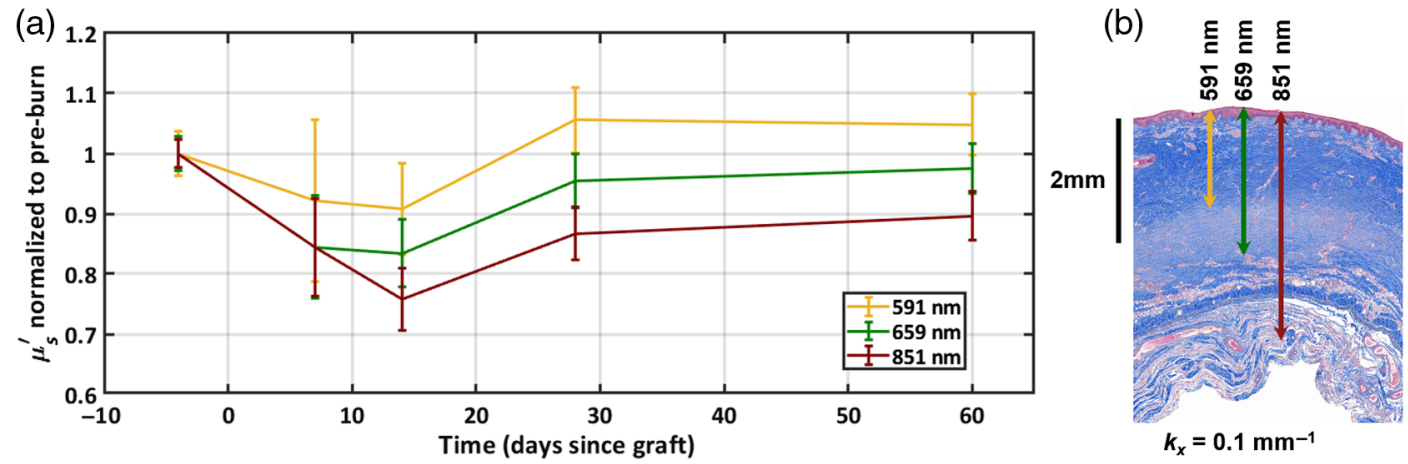

Fig. 8 (a) Mean reduced scattering coefficient at a range of wavelengths of the central $28 \mathrm{~mm}$ of a splitthickness skin graft measured over 60 days after grafting. The error bars represent the standard deviation of the scattering for pixels within the ROI. (b) Masson's histology at D60 showing the penetration depth for a spatial frequency of $k_{x}=0.1 \mathrm{~mm}^{-1}$

(a) No treatment
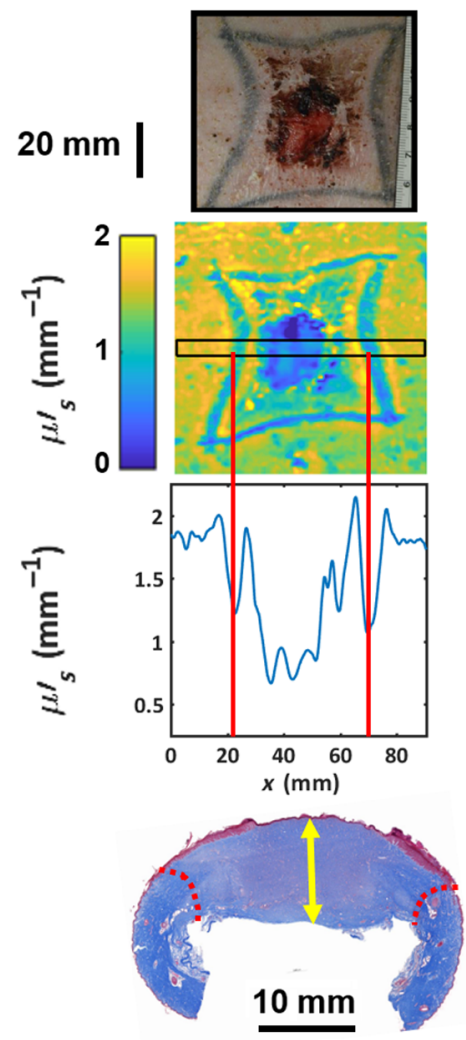

Masson's trichrome histology

Fig. 9. (a) A burn that was surgically debrided but received no graft and (b) a burn that was surgically debrided and received a $1.5: 1$ ratio meshed split-thickness skin graft. Red dotted lines indicate the wound edges. Yellow arrows indicate the granulation tissue that was deposited in the wound bed throughout the time course.

scattering is much higher, which is also reported by the histology and there is minimal wound contraction. The increase in the tattoo size from 60 to $\sim 75 \mathrm{~mm}$ is a result of expansion of the wound periphery after surgical debridement and growth of the animal over the 64-day duration of the study.

In addition to providing a means for mapping changes in tissue structure that are accessible via measurements of the reduced scattering coefficient, SFDI also provides quantitative measurements of the absorption coefficient. The spectra of oxy- and deoxyhemoglobin were fitted to these coefficients at 659 , 731 , and $851 \mathrm{~nm}$ to calculate the concentrations of these chromophores. Figure 10 shows box plots for both the total hemoglobin concentration and the oxygen saturation (oxyhemoglobin concentration/total hemoglobin) for a $3.7-\mathrm{cm}$-diameter central region within the skin graft. We observe increased blood volume after grafting compared to unburned skin; however, the oxygen saturation remains relatively unchanging over the course of 60 days. The increased blood volume may reflect an increase in blood vessel density and/or perfusion arising from increased metabolic demand as the wound heals. The relatively unchanging oxygen saturation suggests that the blood supply is able to provide sufficient oxygen to satisfy the metabolic requirements of the tissue. We are currently investigating this with the addition of laser speckle flow imaging to further elucidate the metabolic state of the healing tissue.

\section{Conclusions}

We have employed SFDI to quantitatively and noninvasively characterize the subsurface changes that occur over the course of burn wound healing of skin in the case of no intervention and following split-thickness skin grafts from 7 to 60 days, using porcine models related to burns, surgical debridement, and skin grafting. SFDI enables us to observe wide-field changes in the reduced scattering coefficient of healing skin, and this correlates with changes observed in the extracellular matrix as reported using conventional histology techniques. Additionally, we have mapped changes in oxy- and deoxyhemoglobin concentrations during healing. Initial results show this has the potential to report on the development of the microvasculature of the wound bed and changes in the metabolism and perfusion of the tissue.

Although SFDI does not yield microscopic resolution and does not offer the contrast that can be achieved through the wide range of stains available for histology, it is noninvasive and has the potential to provide near real-time wide-field maps of optical properties of skin and wounds on a millimeter spatial scale. We, therefore, anticipate that SFDI may prove to be a compelling new approach for noninvasively quantifying the effectiveness of various skin repair interventions to aid the wound healing process. Although our various collaborators, including co-authors for this manuscript, are primarily interested in the application of SFDI to assist in the evaluation of new tissue regenerative strategies within a preclinical framework, we can envision that SFDI (with additional investigation) may also find some utility within the context of informing the process of 


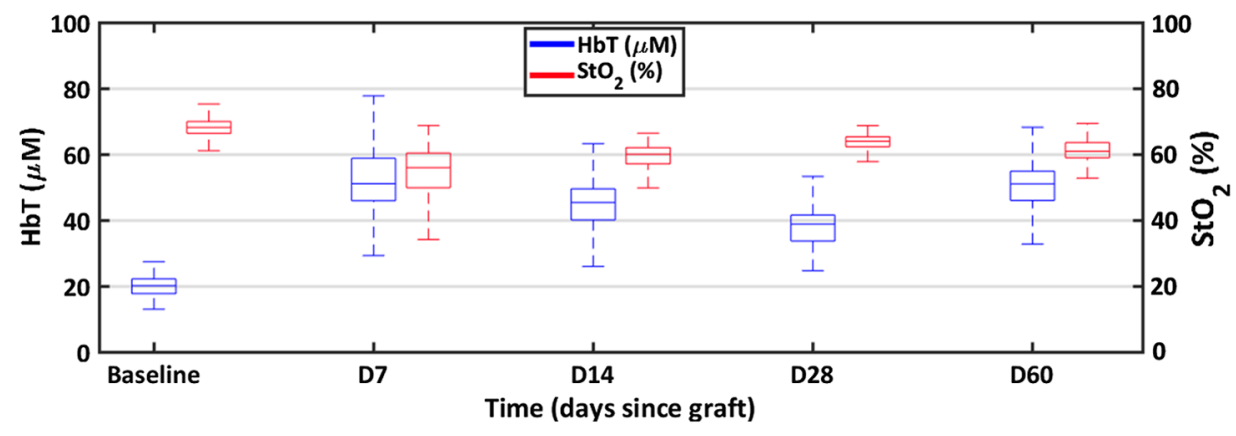

Fig. 10 Evolution of total hemoglobin $(\mathrm{HbT})$ and oxygen saturation $\left(\mathrm{StO}_{2}\right)$ for a burn wound receiving a split-thickness skin graft.

selecting areas within burn tissue that warrant grafting. ${ }^{21}$ There may also be opportunity to employ the device for evaluating the viability of grafts in human subjects.

\section{Disclosures}

A. J. D. is a co-founder of Modulated Imaging Inc., however, he does not participate in the operation of the company. These results have not been shared with MI Inc. prior to journal submission. A. J. D. is in compliance with NIH and UCI conflicts of interest policy. Otherwise, the authors have no financial interests or other conflicts of interest in this manuscript. R. J. C. is an employee of the U.S. federal government, and this work was prepared as part of his official duties.

\section{Acknowledgments}

The authors acknowledge support from the NIH/NIGMS Grant No. R01GM108634 related to SFDI imaging of 3-cm burns in porcine model. In addition, the imaging of the $5-\mathrm{cm}$ burns and skin grafts was supported in part by the Air Force Office of Scientific Research under Award No. FA9550-17-1-0193. We also thank NIH/NIBIB P41EB015890 (a biomedical technology resource) and the Arnold and Mabel Beckman Foundation. This research was also supported in part by an appointment to the Postgraduate Research Participation Program at the U.S. Army Institute of Surgical Research administered by the Oak Ridge Institute for Science and Education through an interagency agreement between the U.S. Department of Energy and USAISR. This research was partly funded by the U.S. Army Medical Research and Materiel Command. The opinions and assertions contained herein are the private views of the authors and are not to be construed as official or reflecting the views of the Department of Defense, Department of Army, or the United States Air Force.

\section{References}

1. American Burn Association, "Burn Incidence fact sheet" (2016).

2. Z. Janzekovic, "A new concept in the early excision and immediate grafting of burns," J. Trauma 10, 1103-1108 (1970).

3. L. Jewell et al., "Rate of healing in skin-grafted burn wounds," Plast. Reconstr. Surg. 120(2), 451-456 (2007).

4. R. Stone et al., "Advancements in regenerative strategies through the continuum of burn care," Front. Pharmacol. 9, 672 (2018).

5. D. M. Burmeister et al., "Utility of spatial frequency domain imaging (SFDI) and laser speckle imaging (LSI) to non-invasively diagnose burn depth in a porcine model," Burns 41(6), 1242-1252 (2015).
6. A. M. Hosking et al., "Spatial frequency domain imaging for burn wound assessment: a case series," Laser Surg. Med. 50, 13-19 (2018).

7. J. Q. Nguyen et al., "Spatial frequency domain imaging of burn wounds in a preclinical model of graded burn severity," J. Biomed. Opt. 18(6), 066010 (2013).

8. A. Ponticorvo et al., "Quantitative long-term measurements of burns in a rat model using spatial frequency domain imaging (SFDI) and laser speckle imaging (LSI)," Laser Surg. Med. 49(3), 293-304 (2017).

9. A. Ponticorvo et al., "Quantitative assessment of graded burn wounds in a porcine model using spatial frequency domain imaging (SFDI) and laser speckle imaging (LSI)," Biomed. Opt. Express 5(10), 3467-3481 (2014).

10. A. Mazhar et al., "Noncontact imaging of burn depth and extent in a porcine model using spatial frequency domain imaging," J. Biomed. Opt. 19(8), 086019 (2014).

11. T. T. A. Nguyen et al., "Novel application of a spatial frequency domain imaging system to determine signature spectral differences between infected and noninfected burn wounds," J. Burn Care Res. 34(1), 44-50 (2013).

12. P. Ghassemi et al., "A polarized multispectral imaging system for quantitative assessment of hypertrophic scars," Biomed. Opt. Express 5(10), 3337-3354 (2014).

13. P. Martin-Mateos et al., "Remote diffuse reflectance spectroscopy sensor for tissue engineering monitoring based on blind signal separation," Biomed. Opt. Express 5(9), 3231-3237 (2014).

14. D. J. Cuccia et al., "Quantitation and mapping of tissue optical properties using modulated imaging," J. Biomed. Opt. 14(2), 024012 (2009).

15. D. J. Cuccia et al., "Modulated imaging: quantitative analysis and tomography of turbid media in the spatial-frequency domain," Opt. Lett. 30(11), 1354-1356 (2005).

16. F. Ayers et al., "Fabrication and characterization of silicone-based tissue phantoms with tunable optical properties in the visible and near infrared domain," Proc. SPIE 6870, 687007 (2008).

17. A. H. Carlsson et al., "Antecedent thermal injury worsens splitthickness skin graft quality: a clinically relevant porcine model of fullthickness burn, excision and grafting," Burns 43(1), 223-231 (2017).

18. D. M. Burmeister et al., "Delivery of allogeneic adipose stem cells in polyethylene glycol-Fibrin hydrogels as an adjunct to meshed autografts after sharp debridement of deep partial thickness burns," Stem Cell Transl. Med. 7(4), 360-372 (2018).

19. A. Ponticorvo et al., "Assessing the predictive capability of optical imaging techniques, spatial frequency domain imaging (SFDI) and laser speckle imaging (LSI), to the gold standard of clinical assessment in a controlled animal model," Proc. SPIE 10467, 104670C (2018).

20. S. T. Horan et al., "Recovery of layered tissue optical properties from spatial frequency-domain spectroscopy and a deterministic radiative transport solver," J. Biomed. Opt. 24(7), 071607 (2018).

21. A. Ponticorvo et al., "Evaluating clinical observation versus spatial frequency domain imaging (SFDI), laser speckle imaging (LSI) and thermal imaging for the assessment of burn depth," Burns 45(2), 450-460 (2019).

Biographies of the authors are not available. 